\title{
No evidence for adaptive sex ratio variation in the cooperatively breeding meerkat, Suricata suricatta
}

\author{
K.J. MacLeod ${ }^{a, *}$ T.H. Clutton-Brock ${ }^{a, b}$ \\ ${ }^{a}$ Large Animal Research Group, University of Cambridge, Cambridge, U.K. \\ ${ }^{\mathrm{b}}$ Mammal Research Institute, University of Pretoria, Pretoria, Republic of South Africa \\ *Correspondence: K. J. MacLeod, Department of Zoology, University of Cambridge, Downing Street, Cambridge \\ CB2 3EJ, U.K. \\ E-mail address: kjm54@cam.ac.uk (K.J. MacLeod).
}

Where a maternal trait influences the fitness of sons or daughters, mothers would be expected to bias the sex ratio towards the sex whose fitness they are more able to increment. In many polygynous species, maternal characteristics affect the fitness of sons more than that of daughters, but, in meerkats, variance in female reproductive success exceeds variance in male reproductive success and maternal rank affects the success of daughters more than sons. Dominant females would therefore be expected to produce an excess of daughters, a reversal of the hypothesis' usual predictions. In a long-term data set, despite a strong effect of maternal rank on daughters' success, we found no indication that dominant females produce female-biased litters. Offspring sex ratios did not deviate significantly from equality, and were also unaffected by maternal mass, age or number of previous litters produced in the same season. We suggest that potential advantages to both mother and offspring of producing and developing in mixed litters may result in the adaptive maintenance of an equal offspring sex ratio. 
Key words: maternal dominance, meerkat, offspring sex ratio variation, Suricata suricatta

When the reproductive value of either sons or daughters is more positively affected by maternal or environmental traits, mothers would be expected to adjust the sex ratio of their offspring to maximize returns to their own fitness (Trivers \& Willard 1973; Charnov 1982). In particular, traits that are related to the capacity of mothers to invest are likely to have stronger effects on the fitness of whichever sex of offspring is more subject to intrasexual competition (Trivers \& Willard 1973). Studies have shown correlations between vertebrate maternal characteristics likely to influence the fitness of offspring and offspring sex ratios, which in some species have been shown to be affected by maternal body condition (CluttonBrock \& Iason 1986; Gosling 1986; Kojola \& Eloranta 1989; Fisher 1999; Nager et al. 1999; Kalmbach et al. 2001) and other condition-related parameters such as age (Blank \& Nolan 1983; Saltz 2001), parity (Huck et al. 1988; Cockburn 1990; Creel et al. 1998) and social rank (Meikle et al. 1984, 1996; Pratt \& Lisk 1989; Cassinello \& Gomendio 1996; Schino 2004; Sheldon \& West 2004).

Mothers of high social rank may be able to pass on benefits to their offspring that result in increased offspring fitness, including rank (Holekamp \& Smale 1991; Hofer \& East 2003; East et al. 2009). This silver-spoon effect (Grafen 1988) may be more likely to affect the fitness of sons or daughters if, for example, variance in reproductive success is higher in one sex than in the other. In these cases, biasing production towards offspring of the sex most subject to reproductive skew, and therefore more likely to be positively affected by maternal status or condition, will increase maternal fitness returns (Trivers \& Willard 1973). For example, maternal rank in red deer, Cervus elaphus, strongly affects the lifetime reproductive success of male offspring, which compete for mating opportunities; as expected, socially 
dominant females produce more male offspring in line with the Trivers-Willard hypothesis (Clutton-Brock et al. 1984). In baboons and macaques, philopatric daughters inherit their mother's rank in stable matrilineal hierarchies where rank correlates with breeding success and skew is high; consequently high-ranking females produce more daughters that are themselves likely to be high-ranking and successful breeders (Altmann 1980; Simpson \& Simpson 1982; Silk 1993). (However, this effect is not ubiquitous in species showing rank inheritance, as there is no sex ratio bias without facultative siblicide in the spotted hyaena, Crocuta crocuta, Wachter et al. 2002.)

The overproduction of daughters by high-ranking mothers when variance in reproductive success is higher among females is in line with models that have shown that the expectations of the Trivers-Willard hypothesis may be reversed if daughters are of higher reproductive value, and if their quality may be influenced by maternal traits (Leimar 1996). Kalahari meerkats, like baboons and macaques, display female philopatry and stable dominance hierarchies where social rank strongly affects reproductive success (Doolan \& MacDonald 1997; Clutton-Brock et al. 1999). Studies of meerkats show that females are subject to higher levels of reproductive skew than males, and that daughters of dominant females are more likely to become dominant and breed successfully themselves than those born to subordinate females (Clutton-Brock et al. 2006; Hodge et al. 2008). On the basis of Leimar's (1996) model, one could predict that high-ranking female meerkats should therefore bias the sex ratio of their offspring towards females whose success is likely to be influenced by their mother's rank, a reversal from the usual expectations of the Trivers-Willard hypothesis.

In this study, we investigated whether maternal status affects the sex ratios of offspring born to wild Kalahari meerkats. These meerkats live in stable groups of up to 50 animals, in which a behaviourally dominant female monopolizes breeding opportunities, 
although subordinate individuals may occasionally breed (Doolan \& MacDonald 1997; Clutton-Brock et al. 1999). The average dominance tenure of dominant females is almost twice as long as that of males (Clutton-Brock et al. 2006), resulting in a monopoly that allows dominant females to produce around 8.7 times as many litters as each subordinate individual (Griffin et al. 2003). This reproductive skew leads to intense intrasexual competition and high levels of variance in reproductive success among females, which are philopatric and compete for dominance and breeding opportunities within their natal groups (Clutton-Brock et al. 2001a, 2006; Griffin 2003). The dominant female maintains her dominant position by aggressively suppressing breeding attempts by subordinate females; these nonbreeding helpers help to rear the offspring of the dominant female (Doolan \& MacDonald 1997; Clutton-Brock et al. 1998, 1999, 2001b). Although subordinate males may be natal or immigrant, both sexes contribute to the rearing of pups born to the dominant female with no effect of kinship (Clutton-Brock et al. 2001b). Females are the more helpful sex, however: as nonbreeding helpers, females contribute consistently more than males to the babysitting and provisioning of pups born to the dominant female (Clutton-Brock et al. 2001b, 2002, 2006). Dominance acquisition is predicted by mass only in females; as offspring born to dominant females are significantly heavier at independence than those born in rare breeding attempts by subordinate females, daughters of dominant females are more likely to attain dominance (Russell et al. 2003; Hodge et al. 2008). Male meerkats disperse and breed outside their natal group and their success in acquiring dominance in groups into which they immigrate is predicted by age, not mass (Spong et al. 2008); therefore it may be assumed that the benefits of increased mass associated with offspring born to dominant females are less likely to affect the fitness of sons.

In the meerkat, high levels of variance in reproductive success, the positive effects of maternal rank on the rank (and therefore reproductive success) of female offspring, and sex 
differences in cooperative behaviours indicate that offspring sex ratio bias may be seen in this species in line with the predictions of a number of sex allocation theories. To date there has been little investigation of adaptive sex ratio adjustment in cooperative mammals (Russell \& Lummaa 2009) or in species in which sex differences in reproductive variance are reversed from the usual expectations. Thus the meerkat provides an interesting system in which to test the following predictions using long-term data. First, because maternal dominance has the potential to affect strongly the breeding success of daughters, dominant females should produce female-biased litters in line with the Trivers-Willard hypothesis (Trivers \& Willard 1973). Second, because females are the more helpful sex, the overall sex ratio of offspring should be biased towards daughters following the local resource enhancement model (Emlen 1986). Third, because levels of within-group competition between females over breeding are high, maternal age should also affect litter sex ratios, which ultimately will affect group sex ratio. Dominant female meerkats live, on average, 4.5 years (Sharp \& Clutton-Brock 2010); younger females should produce an excess of males to reduce female competition, and older females should produce more females with which they will not live to compete, following the local resource competition model (Clark 1978).

\section{METHODS}

\section{Study system and data collection}

Data were collected at the Kuruman River Reserve in the Kalahari region of South Africa $\left(26^{\circ} 58^{\prime} \mathrm{S}, 21^{\circ} 49^{\prime} \mathrm{E}\right)$ between January 1998 and April 2011. Details of climate and habitat are described elsewhere (Russell et al. 2002). All data collection was carried out in accordance with the guidelines of the University of Cambridge and the University of Pretoria. 
The Northern Cape Conservation Authority in South Africa provided permission to carry out the research.

The study population observed over this period included over 40 social groups. All individuals were habituated to close human proximity $(<1 \mathrm{~m})$, and could be identified by unique dye-mark patterns which were applied and maintained while the animal rested without the need for capture (Hodge et al. 2008). Upon visits every 1-3 days the majority of individuals ( $>95 \%)$ were able to be weighed on electronic scales $( \pm 1 \mathrm{~g})$ using small crumbs of hardboiled egg as enticement (Clutton-Brock et al. 2004). Female dominance status could be readily identified, as the dominant female is the primary breeder in the group (CluttonBrock et al. 2001a; Griffin et al. 2003) while all subordinate females are behaviourally submissive to her (O'Riain et al. 2000). Pregnancies were easily detectable in their later stages owing to noticeable increase in mass, and birth dates could be identified (to within 3 days) owing to substantial weight loss.

Meerkat litters are born and remain in an underground burrow until they are approximately 3 weeks old, after which they emerge and begin to forage with the group (Russell et al. 2002). Ultrasonic imaging data has confirmed that few pups are lost during the birth-emergence period during which they remain below ground, unless the whole litter is lost or killed (Russell et al. 2003). Of pups born to dominant females $85 \%$ survived to emergence, and in those litters (17\% of all litters) in which only a proportion of the litter emerged, a mean of 0.6 pups were lost per litter (Russell et al. 2003; A.F. Russell, unpublished data). Although infanticide is common, especially of litters born to subordinate females, selective infanticide has never been observed, and so is not likely to affect litter sex ratios during this period (Young \& Clutton-Brock 2006). Litter size and sex ratio at emergence were therefore judged to be suitable proxies for the same measurement at birth. 
Sex ratio was defined as the proportion of male offspring in the litter (male offspring within litter/total offspring within litter). Pup sex may be determined by examination of the genital region at the age of around 4 weeks when clear sex differences develop, and is confirmed by capture. A quarter of the litters in the data set (93 of 371) contained individuals that died before they were sexed, consistent with the high estimates of preweaning mortality quoted by Hodge et al. (2008) and Russell et al. (2003). However, there is no indication that mortality in the period between emergence and weaning is a reflection of levels of preemergence mortality, as during this period pups leave the burrow and begin to forage (Russell et al. 2002) and are at high risk of predation or separation from the group. Of 1969 individuals that emerged between October 1993 and November 2010, 81.4\% survived to 6 weeks, approximately the age at which pups are weaned, and of those that died, the majority (67.\%) were recorded as having been lost to predation, which is unlikely to result in a sex bias (K.J. MacLeod, unpublished data). Thus, there is no reason to expect that juvenile mortality is sex-biased. Although calculating sex ratios from the remaining pups would not affect the variance of sex ratios, we decided to exclude such litters from all analyses on the grounds that the sample size remained substantial, and although analysis of overall variance would not be biased by random mortality, more valid conclusions could be drawn about the effects of maternal factors on litter sex ratios where the sex ratios were definitively known.

\section{Sex differences in emergence weight}

Consistent sex differences in weights at emergence could indicate a difference in the costs of producing males and females, which would be likely to affect directions of sex ratio bias and the optimal litter sex ratio for each female. Furthermore, sex differences in weight according to maternal status would show that there is a likelihood that meerkat mothers of 
different rank may favour one sex over the other in terms of investment of resources, not number of individual offspring.

Pup weight at emergence was fitted as an explanatory variable in a linear mixed model defined using the lme4 package (Bates et al. 2011) in R 2.12.2 (R Development Core Team 2011) with a Gaussian error distribution, after determining that the data were distributed normally. Litter was included as a random term. Data were available from this data set on 738 pups. Weight at emergence was calculated as the mean of all morning weights taken before a pup was a month old. For each pup, there was an average of $2.99 \pm 1.83$ morning weights available during this period. As lme4 does not report $P$ values, we report estimates, SEs and $t$ values.

\section{Overall variation in litter sex ratios}

The data set contained 278 litters born in 24 groups. We used chi-square tests to compare the observed frequencies of males in each litter size with the frequencies expected under a binomial distribution. For the chi-square approximation to be valid, expected frequencies must be at least 5 . As the sample sizes of litters containing more than four pups were too small for expected male pup frequencies to meet this assumption, it was necessary to group frequencies of males in litter sizes of five and above as follows: in litter sizes of five $(N=52)$, fewer than two males, two, three, more than three males; in litter sizes of $\operatorname{six}(N=21)$, fewer than three males, three, more than three males. This procedure reduced the degrees of freedom for tests on these litter sizes accordingly. Male frequencies in litter sizes of one to four did not need to be grouped. Litter sizes of seven $(N=5)$ were excluded from the analysis as sample sizes were too small even when male frequencies were grouped. Mean sex ratios for each litter size were calculated, and are reported with SDs. 
We estimated the significance of overall deviation from an equal sex ratio using the Monte Carlo significance test method (Mayhew \& Pen 2002). We simulated 100000 new data sets using the observed distribution of litter sizes $(N)$, generating each time a random number of males drawn from a binomial distribution, assuming that the probability of producing a male $(P)$ remained constant and was equal to the observed mean sex ratio. We then calculated the goodness-of-fit statistic ([observed frequency-expected frequency $]^{2} /$ expected frequency, usually associated with the chi-square distribution) for the observed and expected number of litters of each size with each possible number of males. The proportion of simulated distributions with sex ratio variance greater than that observed allows an estimation of the significance of the deviation of the observed from the expected variance in litter sex ratios.

\section{Maternal status and litter sex ratio}

Because the sample size of litters born to dominant females $(N=214)$ far exceeds the sample of litters produced by subordinate females $(N=64)$, we first investigated whether there was a difference in litter sex ratios according to maternal dominance status, and separately analysed the effects of other factors relating to maternal condition on litters conceived during a period of dominance only. Breeding attempts by subordinate females are opportunistic and rarely successful (Griffin et al. 2003); consequently the expectations of adaptive benefits derived from biasing litter sex ratios in these circumstances are greatly reduced. This data set excludes a very small number of litters born to subordinate females that became dominant later in life $(N=14)$. Ideally these litters would have been treated separately, but the sample size was too small to provide sufficient statistical power or a valid comparison to litters from the same females during their period of dominance. 
Maternal dominance status at the time of litter conception was fitted as an explanatory variable in a generalized linear mixed model (GLMM), defined using the lme4 package (Bates et al. 2011) in R 2.12.2 (R Development Core Team 2011) with a logit link function and a binomial error distribution. Conception date could be estimated by backdating 70 days from birth, according to the average meerkat gestation period (Russell et al. 2002). Female dominance status at this time could be readily identified, as the dominant female is the primary breeder in the group (Clutton-Brock et al. 2001a; Griffin et al. 2003) while all subordinate females are behaviourally submissive to her (O'Riain et al. 2000). To prevent pseudoreplication from the nonindependence of multiple litters from the same mother, and to account for between-year variation in seasonal conditions, mother identity and calendar year were included as random terms.

\section{Factors affecting sex ratios of dominant females' litters}

Litter sex ratio was fitted as the response variable in a series of generalized linear mixed models to investigate potential correlations with maternal condition (age and mass at conception, and number of litters previously produced in the same season), litter size and other ecologically important factors (the number of adult helpers within the group, the sex ratio of helpers and rainfall). Maternal mass at conception was calculated as the mean of all mass records taken before foraging started in the morning in the week after the conception date. In a small number of cases the gestation period is shorter than the average of 70 days (S. Sharp, personal communication); because interbirth intervals are also commonly short, using weights from the week after conception (during which there is no discernible pregnancyrelated weight gain) instead of the week before reduced the risk of including weights recorded while the female was still pregnant with a previous litter. Age was measured in days from the date of birth to the date of conception. Litter size was included in the analysis as there is evidence from other species that sex ratio adjustment may vary according to litter size 
(Frank 1990), and was measured at emergence. Total rainfall (mm) in the month prior to conception was included in the model to control for environmental variation. To control for social factors, the number of adult helpers within the group (group member over 6 months of age) was also included in the model. Helper sex ratio (proportion of helpers that were male) was also included, as male and female helpers invest differently in pup-rearing behaviours (Clutton-Brock et al. 2001b); stochastic variation in helper sex ratio may also affect the reproductive value of sons and daughters. Mother identity was included as a random term to prevent problems of pseudoreplication from nonindependence of multiple litters born to the same female.

Preliminary analysis showed that both age and body mass at conception showed suitably high variance to expect significant effects on the sex ratio: in this data set, the average age of dominant females at conception was $1713.15 \pm 661.1$ days. Average body mass at conception showed similarly high levels of variance, with a mean of $768.98 \pm 69.64$. Assessment for collinearity between variables showed that mass and age at conception were positively correlated $(r=0.21, P<0.005)$. To allow the inclusion of both variables in the model we replaced mass in all models with the residuals of the age/mass correlation; this removed the correlation. All input variables were standardized to allow for the direct comparison of parameter estimates by scaling and mean-centering using the arm package in $\mathrm{R}$ (Gelman et al. 2009). All possible interactions were tested separately in individual models with the same random term. Only one had a significant effect: the interaction of helper number and helper sex ratio. This interaction was deemed to be biologically significant, as a skew in helper sex ratio may have stronger effects depending on the total number of helpers, and so was included in the final global model. 
The explanatory variables detailed above produced a total of 160 possible candidate models that were defined using the lme4 package (Bates et al. 2008), all with a logit link function and a binomial error distribution. These models were compared using the $\mathrm{AIC}_{\mathrm{c}}$ (the second-order Akaike information criterion), with the lowest $\mathrm{AIC}_{\mathrm{c}}$ value indicating the best model fit (Akaike 1974). A subset of models was generated by calculating the difference between each model's $\mathrm{AIC}_{\mathrm{c}}$ value and that of the best-fitting model, and using a cutoff of 2 $\mathrm{AIC}_{\mathrm{c}}$ as the criterion for inclusion in the subset. This subset of 15 best-fitting models was then averaged using the MuMIN package (Bartón 2009), combining the parameter estimates of all models relative to their weight (Burnham \& Anderson 2002; following Grueber et al. 2011). This information-theoretic approach allows for the comparison of biologically plausible models representing potential hypotheses and the formation of predictions based on parameter estimates from a number of models, rather than attaching significance values to individual covariates (Burnham \& Anderson 2002). This approach is deemed to have fewer limitations when applied to observational data than null hypothesis testing and model selection by stepwise regression (Burnham et al. 2000).

\section{RESULTS}

Litters ranged in size from one to seven, with a mean of $3.88 \pm 1.25$. Pups emerged $17.58 \pm 3.76$ days after birth, and were weaned at, on average, $50.25 \pm 12.99$ days old $(N=278)$. Sexes were confirmed upon capture when pups were a mean of $27.25 \pm 5.77$ days old $(N=261$, some capture records unavailable). Pup survival to weaning in these litters was $81.3 \%$, and $68.7 \%$ of pup deaths were attributed to predation, consistent with survival and predation proportions for the whole data set (see Methods). 
Sex differences in emergence weight

Pup weight at emergence was not affected by sex (linear mixed model: $\beta \pm \mathrm{SE}=0.56$ $\pm 6.23, t=0.74)$, litter size $(\beta \pm \mathrm{SE}=-1.63 \pm 1.58, t=-1.03)$ or maternal status $(\beta \pm \mathrm{SE}=-7.17$ $\pm 4.21, t=-1.70)$.

\section{Overall variation in litter sex ratios}

The proportion of male offspring in these litters varied between 0 and 1 with a mean sex ratio of $0.53 \pm 0.27, N=278$ (Fig. 1). Analyses of the difference from an equal sex ratio in each litter size class showed no significant departures in each from an equal sex ratio (Table 1). The simulation showed no significant deviation of the observed from the expected variance in litter sex ratios (Monte Carlo test: $x=0.88, P=0.57_{38}$ ). There was insufficient evidence to reject the null hypothesis.

Table 1. Sex ratios (proportion of males) in litters of different size tested for deviation from an equal sex ratio

\begin{tabular}{llllll}
\hline Litter size & $N$ & Mean sex ratio \pm SD & $\chi^{2}$ & $P$ & $d f$ \\
2 & 28 & $0.50 \pm 0.36$ & 0.00 & 0.99 & 2 \\
3 & 60 & $0.5 \pm 0.28$ & 2.76 & 0.43 & 3 \\
4 & 103 & $0.53 \pm 0.26$ & 3.59 & 0.46 & 4 \\
5 & 52 & $0.54 \pm 0.18$ & 5.44 & 0.14 & 3 \\
6 & 21 & $0.52 \pm 0.19$ & 1.59 & 0.45 & 2 \\
7 & 5 & $0.51 \pm 0.22$ & & & \\
\hline
\end{tabular}




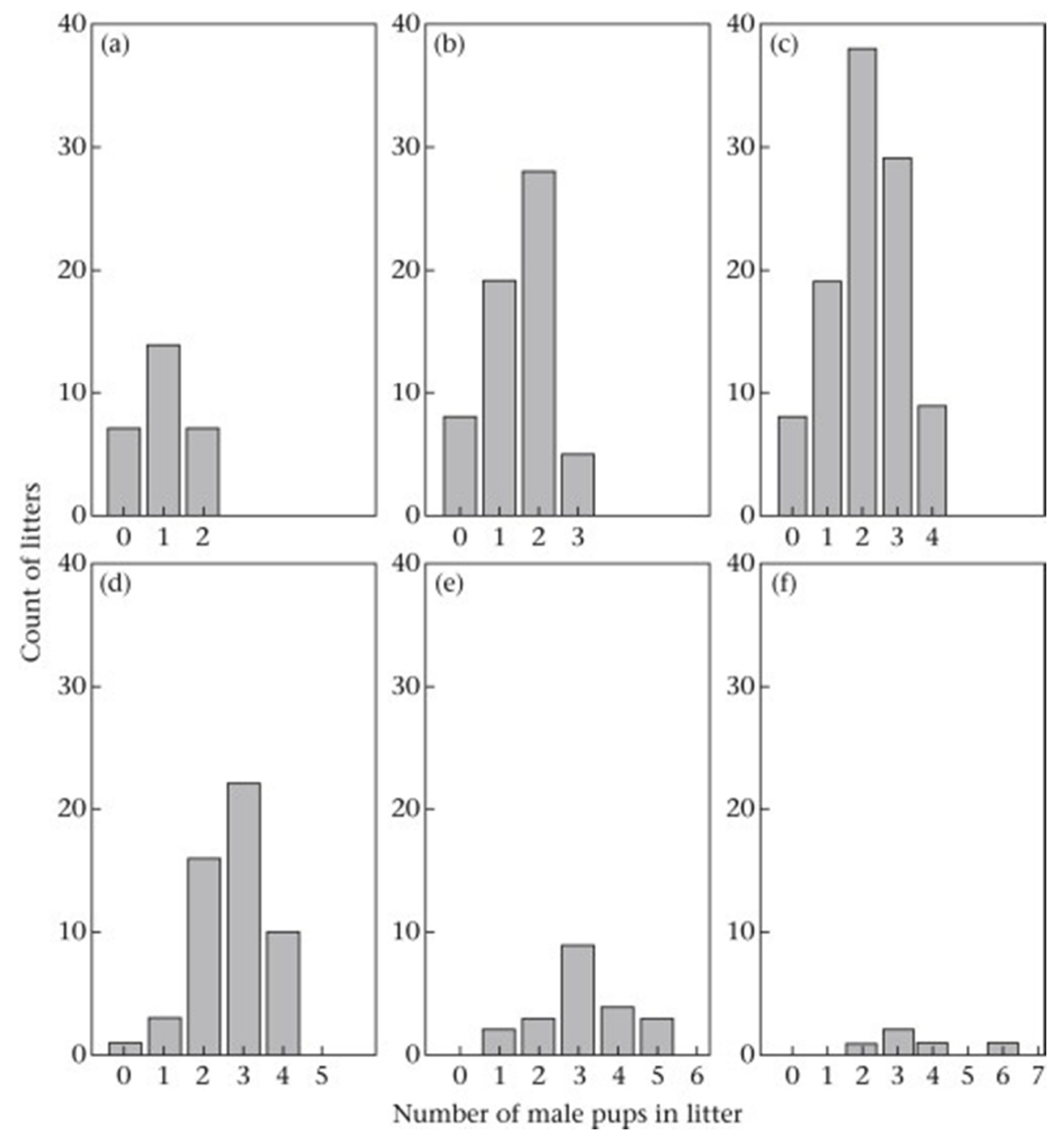

Figure 1. Distribution of litter sex ratios in litters of sizes two to seven.

\section{Maternal status and litter sex ratios}

Of 278 litters, 214 were born to 44 dominant females (each producing a mean of 4.86 \pm 3.56 of these litters) with a much smaller sample of litters born to subordinate females ( $N=64$, each with a mean of $1.16 \pm 0.50$ litters). The mean size of litters born to dominant females $(4.04 \pm 1.20)$ was consistently larger than the mean size of those born to subordinate females $\left(3.33 \pm 1.24\right.$; Mann-Whitney $U$ test: $\left.W=4676, N_{\text {sub }}=64, N_{\text {dom }}=214, P<0.05\right)$. Taking into account mother identity and year of birth, there was no difference in the proportion of male offspring within litters produced by subordinate and dominant females (Table 2). 
Table 2. GLMM investigating the effect of maternal dominance status on litter sex ratio

\begin{tabular}{lllll}
\hline Fixed effect & Estimate & SE & $z$ & $P$ \\
Intercept & -0.09 & 0.16 & -0.57 & 0.57 \\
Status1 & 0.23 & 0.18 & 1.30 & 0.19 \\
\hline
\end{tabular}

Random terms: (1) mother identity: variance $=0.02, \mathrm{SD}=0.15 ;(2)$ year: variance $=0.00, \mathrm{SD}=0.00$. Subordinate litters ('0') were born to females that never achieved dominant breeding positions. Dominant litters (' 1 ') were conceived by females within their dominance tenures.

Factors affecting sex ratios of dominant females' litters

No single factor appeared in all of the best-fitting models, and none were significant in the best-fitting model, although maternal litter order within a season approached significance (Tables 3, 4, Fig. 2). Model averaging ranked the variables in this same order in terms of relative variable importance, and seasonal litter order was ranked the most important (Table 4).

Table 3. $\mathrm{AIC}_{\mathrm{c}}$-ranked candidate model set showing relative importance of maternal mass, age, helper number, helper sex ratio, maternal litter order within season, rainfall and litter size on litter sex ratio in litters born to dominant females

\begin{tabular}{llllll}
\hline Model & Parameters $(k)$ & $\log$ likelihood & $\mathrm{AIC}_{\mathrm{c}}$ & $\Delta_{i}$ & $\omega_{i}$ \\
\hline Seasonal litter order, mass & 4 & -120.88 & 249.95 & 0 & 0.11 \\
Helper number, helper sex & 7 & -117.72 & 249.98 & 0.04 & 0.11 \\
ratio, seasonal litter order, & & & & \\
mass, helper number*helper sex & & & & & \\
ratio & & & & & \\
Helper number, helper sex & 6 & -118.95 & 250.31 & 0.37 & 0.09
\end{tabular}


ratio, seasonal litter order,

helper number*helper sex ratio

Seasonal litter order

Helper number, seasonal litter

order, mass

Age, seasonal litter order, mass

Helper number, seasonal litter

order

Mass

Age, mass

Age, seasonal litter order

Helper sex ratio, seasonal litter

order, mass

Helper sex ratio, helper

number, mass, helper

number*helper sex ratio

Helper sex ratio, helper

number, seasonal litter order,

rainfall, mass, helper

number*helper sex ratio

Litter size, seasonal litter order, 5

mass

Seasonal litter order, rainfall, 5

4

8
$250.53 \quad 0.59$

0.08

$-120.21$

$250.71 \quad 0.76$

0.07

$-120.21$

$250.72 \quad 0.77$

0.07

$-121.33$

$250.84 \quad 0.90$

0.07

$-122.47$

$-121.53$

$-121.59$

$-120.64$

$-119.66$

$251.72 \quad 1.77$

0.05

$-117.59$

$251.89 \quad 1.95$

0.04

$-120.81$

$251.90 \quad 1.96$

mass

Submodels were derived from a global model using a cutoff of $2 \mathrm{AIC}_{\mathrm{c}}$ greater than the best fitting model.

Because of a highly significant correlation between mass and age (days) at conception, mass is replaced with the residuals of the correlation. $\mathrm{AIC}_{\mathrm{c}}$ weights $\left(\omega_{i}\right)$ show the relative strength of support for each model and were used to generate the model-averaged estimates for each parameter. Mother identity $(N=44)$ was included as a random term in each model. 
Table 4. The results of generalized linear mixed models of factors potentially affecting litter sex ratio

\begin{tabular}{|c|c|c|c|c|}
\hline Fixed effect & Estimate & Unconditional SE & \multicolumn{2}{|c|}{ Relative variable importance } \\
\hline Intercept & 0.28 & 0.29 & & \\
\hline Seasonal litter & 0.27 & 0.14 & \multicolumn{2}{|l|}{0.84} \\
\hline \multicolumn{5}{|l|}{ order } \\
\hline Mass & -0.25 & 0.15 & \multicolumn{2}{|l|}{0.70} \\
\hline Helper number & 1.15 & 0.91 & \multicolumn{2}{|l|}{0.43} \\
\hline Helper sex ratio & -0.80 & 0.65 & \multicolumn{2}{|l|}{0.33} \\
\hline Helper & -2.90 & 1.43 & \multicolumn{2}{|l|}{0.29} \\
\hline \multicolumn{5}{|c|}{ number*helper sex } \\
\hline \multicolumn{5}{|l|}{ ratio } \\
\hline Age & 0.18 & 0.15 & \multicolumn{2}{|l|}{0.18} \\
\hline Rainfall & 0.06 & 0.15 & \multicolumn{2}{|l|}{0.08} \\
\hline Litter size & -0.06 & 0.16 & \multicolumn{2}{|l|}{0.04} \\
\hline Best model & Estimate & SE & $z$ & $P$ \\
\hline Intercept & 0.13 & 0.07 & 1.81 & 0.07 \\
\hline Seasonal litter & 0.25 & 0.14 & 1.80 & 0.07 \\
\hline \multicolumn{5}{|l|}{ order } \\
\hline Mass & -0.24 & 0.15 & -1.63 & 0.10 \\
\hline
\end{tabular}



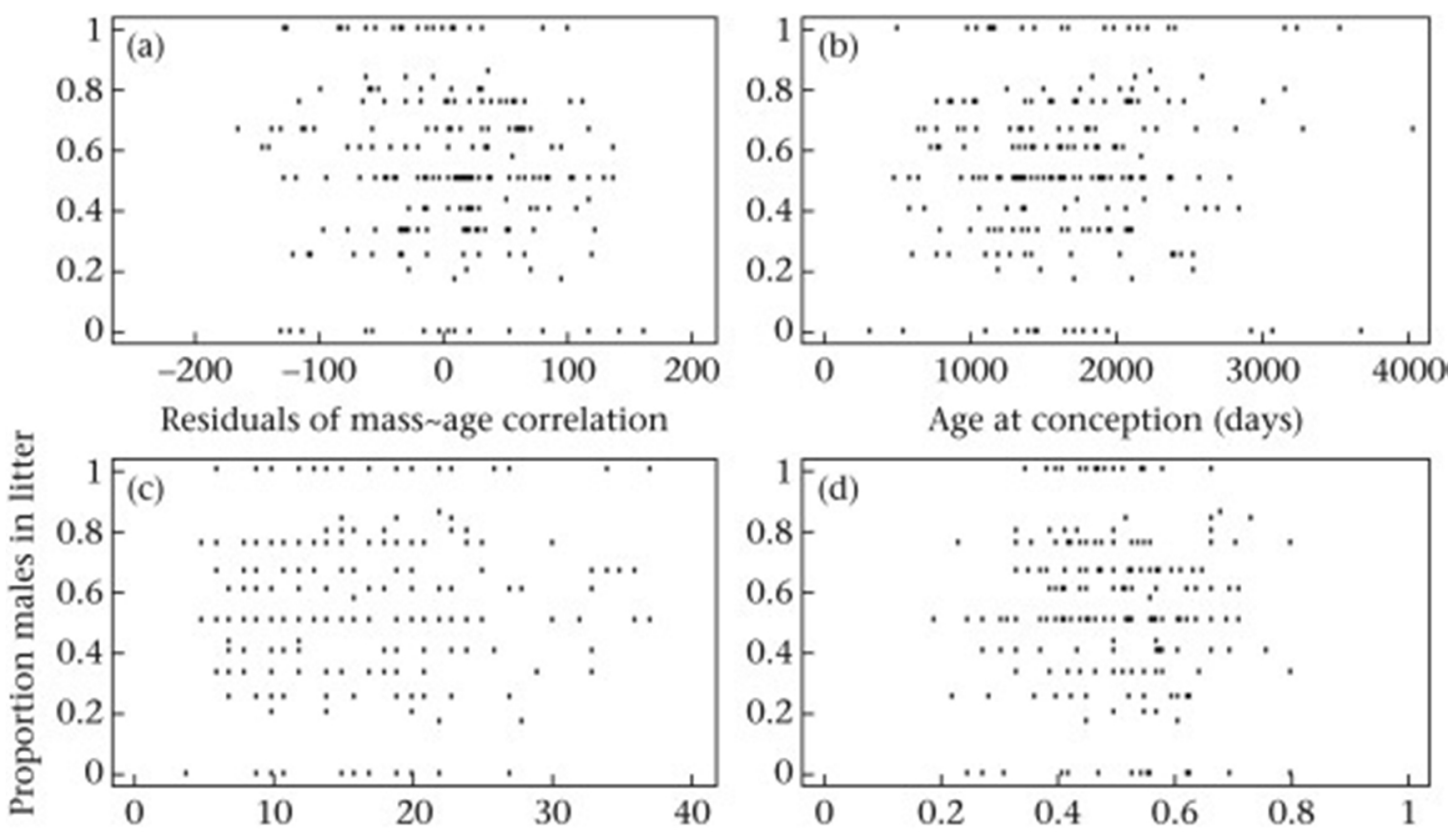

Age at conception (days)

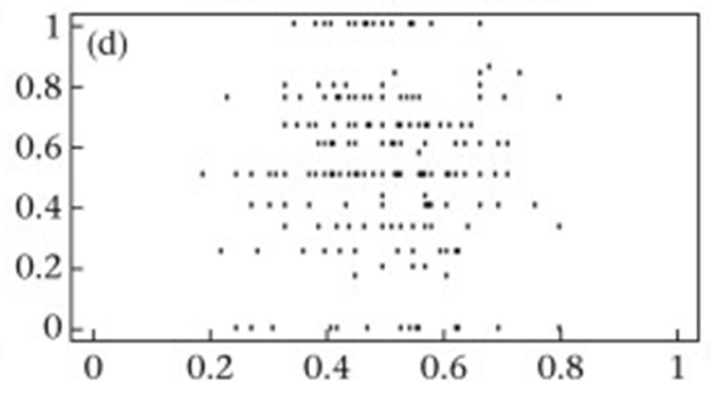

Adult helpers present at conception
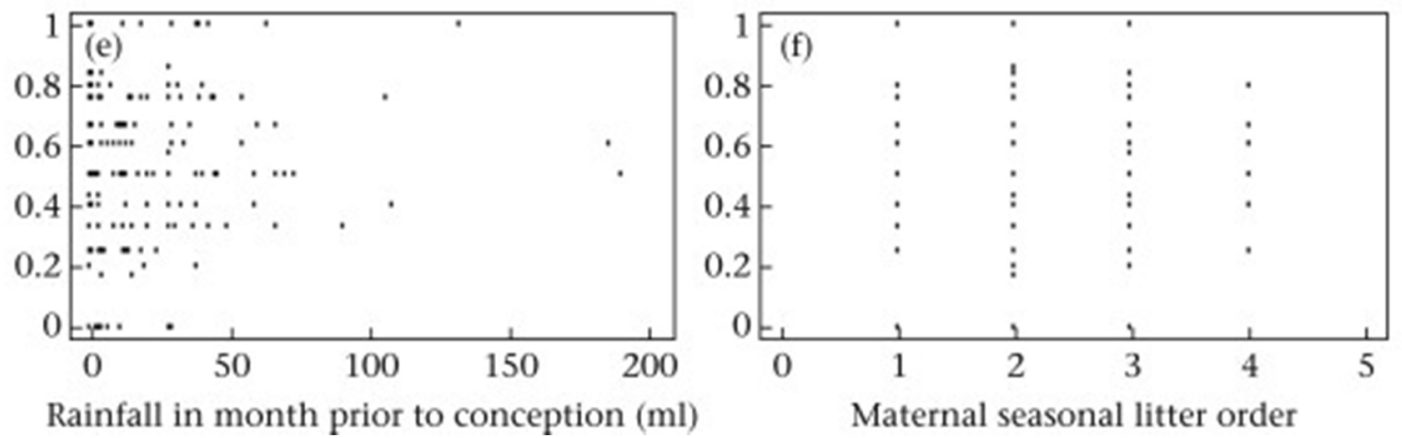

Rainfall in month prior to conception (ml)

Maternal seasonal litter order

Figure 2. Factors affecting litter sex ratio in litters born to dominant females. Mass at conception is replaced with the residuals of the mass-age correlation, providing a measure of whether the individual was heavier or lighter than predictions based on the linear relationship $(r=21)$.

\section{DISCUSSION}

Despite predictions for offspring sex ratio bias, variance in meerkat litter sex ratios did not exceed the binomial variance expected under Mendelian segregation and, overall, offspring sex ratios did not differ from parity. Meerkat mothers did not adjust the sex ratio of their offspring according to their social rank, contradicting predictions that dominant females should produce litters biased towards daughters, which have a better chance of themselves 
becoming high-ranking and consequently breeding successfully. Furthermore, dominant female meerkats did not vary offspring sex ratios according to their mass at conception. Litter sex ratios were also unaffected by nonmaternal factors that could affect chances of success: the number of adult helpers present in the group at conception or rainfall in the month prior to conception. Finally, the hypothesis that litter sex ratios are affected by maternal age was not supported by our models. This is counter to our secondary hypothesis that females may produce an equal sex ratio over their lifetime, but with temporal variation in the frequencies of male and female offspring to avoid reproductive competition with competitive daughters produced early in life.

There are a number of possible explanations for this lack of litter sex ratio variation. Sex ratio adjustment is not always seen where it is expected; for example, maternal traits do not affect offspring sex ratios in Soay sheep, Ovis aries, despite predictions that high-quality mothers should produce males in line with the Trivers-Willard hypothesis (Trivers \& Willard 1973; Lindström et al. 2002). Griffin et al. (2005) argued that sex ratio effects are strongest when the benefits of manipulating sex ratios are highest: the difference in reproductive value of daughters relative to that of sons may be overestimated, reducing the payoffs of sex ratio adjustment, and therefore also reducing the likely strength of any sex ratio effect. Alternatively, producing equal numbers of male and female offspring may be adaptive if there are benefits to offspring of developing in mixed litters (Ryan \& Vandenbergh 2002), or the costs of producing competitive daughters may be matched or outweighed by the benefits derived from their substantial contributions to pup rearing and other cooperative behaviours. Furthermore, a mechanism by which mammals might adjust the sex ratio of their offspring may be lacking, or chromosomal sex determination may constrain sex ratio manipulation too strongly (Williams 1979; West \& Sheldon 2002; Uller et al. 2007). All of these possibilities may apply to meerkats, as discussed below. 
Although daughters of dominant females are more likely to become dominant breeders themselves than the daughters of subordinate females, few such daughters will actually succeed in attaining dominance owing to long dominance tenures and low female dispersal rates (Clutton-Brock et al. 2006), reducing the payoffs of sex ratio adjustment to a point where it is no longer of benefit. Sex ratio bias towards daughters increases the number of candidates for dominance a mother may produce, but winning the competition may require quality, not quantity. Instead of biasing investment towards females in terms of litter sex ratio, mothers may instead bias their investment towards potential future dominant females by directing resources towards a few daughters during gestation (prenatal investment) or during lactation (postnatal investment). Although this study shows that, on average, male and female pups did not differ significantly in weight at emergence according to maternal status, females may benefit from differential investment in offspring of either sex, or in whole litters, at different times both in a season and in their lifetime. Although not significant in our bestfitting model, the order in which litters came in the season was the most important factor in determining offspring sex ratio of those that we tested; this may indicate temporal change in the reproductive value of male and female offspring. Future work will consider what factors influence both pre- and postnatal maternal investment in male and female offspring, taking into account the high levels of helper investment in the form of pup provisioning and allolactation, which will itself provide the basis for further study.

We predicted that because the rank of mothers strongly affects the fitness of daughters they should produce more daughters, but this hypothesis may underestimate the effect of maternal rank on the reproductive success of sons, which, if equally substantial, may further reduce the payoffs of biasing sex ratios towards females. Although dominance acquisition in males is not predicted by mass (Spong et al. 2008), which is influenced by maternal rank (Russell et al. 2003), maternal status may otherwise still positively affect the reproductive 
success of males. In the spotted hyaena, in which females are also philopatric and males disperse to breed, maternal rank influences the breeding success of sons through effects on dispersal patterns and the age at first reproduction: high-born males start reproducing earlier and disperse to groups more likely to offer breeding opportunities (Höner et al. 2010). Similar effects may be seen in the meerkat. Although age predicts the likelihood of dominance acquisition, this is not the only route to breeding success for males, which may gain paternity through opportunistic breeding attempts on extraterritorial forays (Young 2003). Males that were heavier at independence begin prospecting for these breeding attempts earlier (Mares 2012) potentially resulting in increased lifetime reproductive success. Mass may also affect survival to and success of dispersal attempts, or the ability to retain tenure if dominance is acquired. New paternity data from long-term samples of the meerkat population will allow better quantification of reproductive success for males that disperse to groups within the population.

The lack of sex ratio variation in meerkat litters overall suggests a lack of support for the hypothesis that, under a local resource enhancement model, mothers should produce an excess of the helpful sex (Emlen et al. 1986). Equally, the lack of variation according to maternal age also suggests that, despite predictions based on the local resource competition model (Clark 1978), offspring sex ratios are not biased away from the more competitive sex when residual reproductive value remains high. Where the more helpful sex is also the more competitive, a lack of bias may be adaptive; for example in the black-tailed prairie dog, Cynomys ludovicianus, in which, like the meerkat, females are philopatric and cooperate with female kin in long-term cooperative partnerships, but there is also strong competition between females, in this case for burrows (Hoogland 1995). In this case, the local resource enhancement model would predict a bias towards daughters. However, the local resource competition model would predict skewing the sex ratio of offspring away from females, the 
more competitive sex, in this case towards males. As a result, an equal sex ratio is maintained (Hoogland 1995). Overall parity could imply that there is a trade-off between the relative costs and benefits of producing daughters that results in an optimal sex ratio of 1:1.

Producing litters of equal sex ratio may be beneficial, for example if female offspring born in litters containing both sexes benefit from the presence of male fetuses in utero, increasing exposure to prenatal androgens, which can lead to an increase in aggression (Vandenbergh \& Huggett 1994; Staub \& DeBeer 1997; Ryan \& Vandenbergh 2002; Monclús \& Blumstein 2012). Competition for dominant breeding positions is intense among meerkat female peers; consequently aggression is likely to be an important determinant of fitness particularly among females (Clutton-Brock et al. 2006; Hodge et al. 2008).

A prenatal mechanism by which vertebrate females may overcome the constraints of chromosomal sex determination to adjust the sex ratio of their offspring is not conclusively known, and may not exist in all mammal classes (Williams 1979; West \& Sheldon 2002; Uller et al. 2007). Proposed mechanisms based on physiological parameters such as circulating levels of stress hormones or blood glucose, which are likely to correspond to maternal condition or rank, have been associated with bias towards male offspring where maternal stress is high (Cameron 2004). Dominant female meerkats have higher circulating levels of cortisol than subordinate females owing to the physiological stress of dominating group members or to possible alteration of basal steroidogenesis patterns caused by frequent pregnancy (Carlson et al. 2004). This mechanism of sex ratio adjustment would predict that in the meerkat, dominant females would produce sex ratios biased towards male offspring, contradicting our predictions that dominant females should produce daughters. However, the lack of variation in offspring sex ratio would indicate that this mechanism is not operating in the meerkat. Equally, a mechanism favouring the adjustment of offspring sex ratios favouring 
female offspring may not exist in this species, explaining the lack of female bias in litters where it was expected.

The lack of offspring sex ratio variation in the meerkat despite predictions for a female bias in litters born to dominant females lends weight to the growing body of literature indicating that facultative sex ratio adjustment in vertebrates is not a consistent biological phenomenon (Hardy 2002; Krackow 2002; Ewen et al. 2004). Our study is also in agreement with the findings of Brown \& Silk (2002): in large samples, differences in offspring sex ratios produced by females of different rank shrink, and data are distributed as would be expected by chance, indicating a likelihood that effects of rank on litter sex ratios in other species may be a stochastic effect as a result of small sample sizes. Nevertheless, investigation of what benefits may be derived from maintaining an equal sex ratio in species in which bias is not seen but would be expected may be useful, for example in the meerkat and in Soay sheep. Although we do not expect using litter sex ratios at emergence to affect offspring sex ratio variation in this study (see Methods), the inability to ascertain litter sex ratios at birth in the field is a limitation of this data set, which may be solved in the future by conducting more ultrasonic imaging on pregnant females and the potential use of in utero sexing techniques. Also, the potential for underestimating the reproductive success of sons, which disperse out of the population, limits our ability to determine conclusively the difference in reproductive value between sons and daughters of dominant females. In the meerkat system, future analysis of the effects of stochastic variation in litter sex ratios on individual levels of aggression, cooperation and lifetime reproductive success of offspring could illuminate further the relative costs or benefits of producing biased versus unbiased litters, which in turn could shed light on potentially adaptive benefits derived from the maintenance of an equal offspring sex ratio in this species. 
Acknowledgments

Thanks to Marta Manser, all staff, volunteers and students who have contributed to the longterm database at the Kalahari Meerkat Project and to Andrew Bateman for statistical assistance and advice. Comments from Rafael Mares, Sinead English, Stuart Sharp, the Editor and two anonymous referees were very helpful. Advice and encouragement from Dr Jeff Graves, Professor Peter Slater and Dr Athol McLachlan were much appreciated.

\section{REFERENCES}

Akaike, H. 1974. A new look at the statistical model identification. IEEE Transactions on Automatic Control, 19, 716-723.

Alcock, J. 1993. Animal Behavior: an Evolutionary Approach. 5th edn. Sunderland, Massachusetts: Sinauer Associates.

Altmann, J. 1980. Baboon Mothers and Infants. Cambridge, Massachussetts: Harvard University Press.

Bartón, K. 2009. MuMIn: multi-model inference, R package, version 1.7.2: http://cran.rproject.org/web/packages/MuMIn/MuMIn.pdf.

Bates, D., Maechler, M. \& Dai, B. 2008. Linear mixed-effects models using S4 classes, R package, version 0.999375-28: http://lme4.r-forge.r-project.org/.

Blank, J.L. \& Nolan, V. 1983. Offspring sex ratio in red-winged blackbirds is dependent on maternal age. Proceedings of the National Academy of Sciences, U.S.A., 80, 6141-6145.

Brown, G.R. \& Silk, J.B. 2002. Reconsidering the null hypothesis: is maternal rank associated with birth sex ratios in primate groups? Proceedings of the National Academy of Sciences, U.S.A., 99, 11252-11255. 
Burnham, K.P. \& Anderson, D.R. 2002. Model Selection and Multimodel Inference: a Practical Information Theoretic Approach. New York: Springer.

Burnham, K.P., Thompson, W.L. \& Anderson, D.R. 2000. Null hypothesis testing: problems, prevalence, and an alternative. The Journal of Wildlife Management, 64, 912-923. Cameron, E.Z. 2004. Facultative adjustment of mammalian sex ratios in support of the Trivers-Willard hypothesis: evidence for a mechanism. Proceedings of the Royal Society B, 271, 1723-1728.

Carlson, A.A., Young, A.J., Russell, A.F., Bennett, N.C., McNeilly, A.S. \& Clutton-

Brock, T. 2004. Hormonal correlates of dominance in meerkats (Suricata suricatta). Hormones and Behaviour, 46, 141-150.

Cassinello, J. \& Gomendio, M. 1996. Adaptive variation in litter size and sex ratio at birth in a sexually dimorphic ungulate. Proceedings of the Royal Society B, 263, 1461-1466.

Charnov, E.L. 1982. The Theory of Sex Allocation. Princeton, New Jersey: Princeton University Press.

Clark, A.B. 1978. Sex-ratio and local resource competition in a prosimian primate. Science, 201, 163-165.

Clarke, F.M. \& Faulkes, C.G. 1997. Dominance and queen succession in captive colonies of the eusocial naked mole-rat, Heterocephalus glaber. Proceedings of the Royal Society B, 264, 993-1000.

Clarke, F.M., Miethe, G.H. \& Bennett, N.C. 2001. Reproductive suppression in female Damaraland mole-rats Cryptomys damarensis: dominant control or self-restraint? Proceedings of the Royal Society B, 268, 899-909.

Clutton-Brock, T.H. \& Iason, G.R. 1986. Sex-ratio variation in mammals. Quarterly Review of Biology 61, 339-374. 
Clutton-Brock, T., Albon, S. \& Guinness, F. 1984. Maternal dominance, dominance, breeding success and birth sex-ratios in red deer. Nature, 308, 358-360.

Clutton-Brock, T.H., Brotherton, P.N.M., Smith, R., McIlrath, G.M., Kansky, R., Gaynor, D., O'Riain, M.J. \& Skinner, J.D. 1998. Infanticide and expulsion of females in a cooperative mammal. Proceedings of the Royal Society B, 265, 2291-2295.

Clutton-Brock, T.H., Gaynor, D., McIlrath, G.M., MacColl, A.D.C., Kansky, R., Chadwick, P., Manser, M., Brotherton, P.N.M. \& Skinner, J.D. 1999. Predation, group size and mortality in a cooperative mongoose, Suricata suricatta. Journal of Animal Ecology, 68, 672-683.

Clutton-Brock, T.H, Brotherton, P.N.M., O’Riain, M.J., Griffin, A.S., Gaynor, D., Kansky, R., Manser, M.B. \& McIlrath, G.M. 2000. Individual contributions to babysitting in a cooperative mongoose, Suricata suricatta. Proceedings of the Royal Society B, 267, 301305.

Clutton-Brock, T.H., Brotherton, P.N.M., Russell, A.F., O’Riain, M.J., Gaynor, D., Kansky, R., Griffin, A., Manser, M., Sharpe, L., McIlrath, G.M. et al. 2001a. Cooperation, control, and concession in meerkat groups. Science, 291, 478-481.

Clutton-Brock, T.H., Brotherton, P.N.M., O’Riain, M.J., Griffin, A.S., Gaynor, D., Kansky, R., Sharpe, L. \& McIlrath, G.M. 2001b. Contributions to cooperative rearing in meerkats. Animal Behaviour, 61, 705-710.

Clutton-Brock, T.H., Russell, A.F., Sharpe, L.L., Young, A.J., Balmforth, Z. \& McIlrath, G.M. 2002. Evolution and development of sex differences in cooperative behavior in meerkats. Science, 297, 253 -256.

Clutton-Brock, T.H., Russell, A.F. \& Sharpe, L.L. 2004. Behavioural tactics of breeders in cooperative meerkats. Animal Behaviour, 68, 1029-1040.

Clutton-Brock, T.H., Hodge, S.J., Spong, G., Russell, A.F., Jordan, N.R., Bennett, N.C., 
Sharpe, L.L. \& Manser, M.B. 2006. Intrasexual competition and sexual selection in cooperative mammals. Nature, 444, 1065-1068.

Cockburn, A. 1990. Sex-ratio variation in marsupials. Australian Journal of Zoology, 37, 467-479.

Cockburn, A., Scott, M.P. \& Dickman, C.R. 1985. Sex-ratio and intrasexual kin competition in mammals. Oecologia, 66, 427-429.

Creel, S., Creel, N.M. \& Monfort, S.L. 1998. Birth order, estrogens and sex-ratio adaptation in African wild dogs (Lycaon pictus). Animal Reproduction Science, 53, 315-320.

Doolan, S.P. \& Macdonald, D.W. 1997. Breeding and juvenile survival among slendertailed meerkats (Suricata suricatta) in the south-western Kalahari: ecological and social influences. Journal of Zoology, 242, 309-327.

East, M.L., Höner, O.P., Wachter, B., Wilhelm, K., Burke, T. \& Hofer, H. 2009.

Maternal effects on offspring social status in spotted hyenas. Behavioral Ecology, 20, 478483.

Emlen, S.T. 1997. When mothers prefer daughters over sons. Trends in Ecology \& Evolution, 12, 291-292.

Emlen, S.T., Emlen, J.M., \& Levin, S.A. 1986. Sex-ratio selection in species with helpers at the nest. The American Naturalist, 127, 1-8.

Fisher, D.O. 1999. Offspring sex ratio variation in the bridled nailtail wallaby, (Onychogalea fraenata). Behavioral Ecology and Sociobiology, 45, 411-419.

Frank, L.G. 1990. Sex allocation theory for birds and mammals. Annual Review of Ecology and Systematics, 21, 13-55.

Gelman, A., Su, Y., Yajima, M., Hill, J., Pittau, M.G., Kerman, J. \& Zheng, T. 2012. arm: data analysis using regression and multilevel/hierarchical models, R package, version 1.5-02: http://cran.r-project.org/web/packages/arm/. 
Gosling, L.M. 1986. Selective abortion of entire litters in the coypu: adaptive control of offspring production in relation to quality and sex. The American Naturalist, 127, 772-795. Griffin, A.S., Pemberton, J.M., Brotherton, P.N.M., McIlrath, G., Gaynor, D., Kansky, R., O'Riain, J. \& Clutton-Brock, T.H. 2003. A genetic analysis of breeding success in the cooperative meerkat (Suricata suricatta). Behavioral Ecology, 14, 472-480.

Griffin, A.S., Sheldon, B.C. \& West, S.A. 2005. Cooperative breeders adjust offspring sex ratios to produce helpful helpers. American Naturalist, 166, 628-632.

Grueber, C.E., Nakagawa, S., Laws, R.J. \& Jamieson, I.G. 2011. Multimodel inference in ecology and evolution: challenges and solutions. Journal of Evolutionary Biology, 24, 699711.

Hodge, S.J., Manica, A., Flower, T.P., Clutton-Brock, T.H. 2008. Determinants of reproductive success in dominant female meerkats. Journal of Animal Ecology, 77, 92-102. Hofer, H. \& East, M.L. 2003. Behavioural processes and costs of co-existence in female spotted hyenas: a life history perspective. Evolutionary Ecology, 17, 315-331.

Holekamp, K.E. \& Smale, L. 1991. Dominance acquisition during mammalian social development: the 'inheritance' of maternal rank. American Zoologist, 31, 306-317.

Höner, O.P., Wachter, B., Hofer, H., Wilhelm, K., Thierer, D., Trillmich, F., Burke, T. \& East, M.L. 2010. The fitness of dispersing spotted hyaena sons is influenced by maternal social status. Nature Communications, 1, 60.

Huck, U.W., Pratt, N.C., Labov, J.B. \& Lisk, R.D. 1988. Effects of age and parity on litter size and offspring sex ratio in golden hamsters (Mesocricetus auratus). Journal of Reproduction and Fertility, 83, $209-214$.

Kalmbach, E., Nager, R.G., Griffiths, R. \& Furness, R.W. 2001. Increased reproductive effort results in male-biased offspring sex ratio: an experimental study in a species with reversed sexual size dimorphism. Proceedings of the Royal Society B, 268, 2175-2179. 
Kojola, I. \& Eloranta, E. 1989. Influences of maternal body weight, age, and parity on sex ratio in semidomesticated reindeer (Rangifer t. tarandus). Evolution, 43, 1331-1336. Krackow, S. 2002. Why parental sex ratio manipulation is rare in higher vertebrates. Ethology, 108, 1041-1056.

Leimar, O. 1996. Life history analysis of the Trivers and Willard sex ratio problem. Behavioral Ecology, 7, 316-215.

Lindström, J., Coulson, T., Kruuk, L., Forchhammer, M.C., Coltman, D.W. \& CluttonBrock, T.H.2002. Sex-ratio variation in soay sheep. Behavioral Ecology and Sociobiology, 53, 25-30.

Mares, R. 2012. Extraterritorial prospecting and territory defence in cooperatively breeding meerkats. Ph.D. thesis, University of Cambridge.

Mayhew, P.J. \& Pen, I. 2002. Comparative analysis of sex ratios. In:. Sex Ratios: Concepts and Research Methods (Ed. by I. C. W. Hardy), pp. 132-157. Cambridge: Cambridge University Press.

Meikle, D.B., Tilford, B.L. \& Vessey, S.H. 1984. Dominance rank, secondary sex-ratio, and reproduction of offspring in polygynous primates. American Naturalist, 124, 173-188.

Meikle, D.B., Drickamer, L.C., Vessey, S.H., Arthur, R.D. \& Rosenthal, T.L.1996. Dominance rank and parental investment in swine (Sus scrofa domesticus). Ethology, 102, 969-978.

Monclús, R. \& Blumstein, D.T. 2012. Litter sex composition affects life-history traits in yellow-bellied marmots. Journal of Animal Ecology, 81, 80-86.

Nager, R.G., Monaghan, P,. Griffiths, R., Houston, D.C. \& Dawson, R. 1999.

Experimental demonstration that offspring sex ratio varies with maternal condition. Proceedings of the National Academy of Sciences, U.S.A., 96, 570-573. 
O’Riain, M.J., Bennett, N.C., Brotherton, P.N.M., McIlrath, G. \& Clutton-Brock, T.H. 2000. Reproductive suppression and inbreeding avoidance in wild populations of co operatively breeding meerkats (Suricata suricatta). Behavioral Ecology and Sociobiology, 48, 471-477.

\section{R Development Core Team. 2011. R: A Language and Environment for Statistical}

Computing. Vienna: R Foundation for Statistical Computing.

Roff, D.A. 2002. Life History Evolution. Sunderland, Massachusetts: Sinauer Associates. Russell, A.F. \& Lummaa, V. 2009. Maternal effects in cooperative breeders: from hymenopterans to humans. Philosophical Transactions of the Royal Society B, 364, 11431167.

Russell, A.F., Clutton-Brock, T.H., Brotherton, P.N.M., Sharpe, L.L., McIlrath, G.M., Dalerum, F.D., Cameron, E.Z. \& Barnard, J.A. 2002. Factors affecting pup growth and survival in co-operatively breeding meerkats, Suricata suricatta. Journal of Animal Ecology, 71, 700-709.

Russell, A.F., Brotherton, P.N.M., McIlrath, G.M., Sharpe, L.L. \& Clutton-Brock, T.H. 2003. Breeding success in cooperative meerkats: effects of helper number and maternal state. Behavioral Ecology, 14, 486-492.

Russell, A.F., Young, A.J., Spong, G., Jordan, N.R. \& Clutton-Brock, T.H. 2007. Helpers increase the reproductive potential of offspring in cooperative meerkats. Proceedings of the Royal Society B, 274, $513-520$.

Ryan, B.C. \& Vandenbergh, J.G. 2002. Intrauterine position effects. Neuroscience \& Biobehavioral Reviews, 26, 665-678.

Saltz, D. 2001. Progeny sex ratio variation in ungulates: maternal age meets environmental perturbation of demography. Oikos, 94, 377-384. 
Schino, G. 2004. Birth sex ratio and social rank: consistency and variability within and between primate groups. Behavioral Ecology, 15, 850-856.

Sharp, S.P. \& Clutton-Brock, T.H. 2010. Reproductive senescence in a cooperatively breeding mammal. Journal of Animal Ecology, 79, 176-183.

Sheldon, B.C. \& West, S.A. 2004. Maternal dominance, maternal condition, and offspring sex ratio in ungulate mammals. American Naturalist, 163, 40-54.

Silk, J.B. 1993. The evolution of social conflict among female primates. In: Primate Social Conflict (Ed. by W. A. Mason \& S. Mendoza), pp. 49-83. Albany, New York: State University of New York Press.

Simpson, M. \& Simpson, A. 1982. Birth sex-ratios and social rank in rhesus-monkey mothers. Nature, 300, 440-441.

Spong, G.F., Hodge, S.J., Young, A.J. \& Clutton-Brock, T.H. 2008. Factors affecting the reproductive success of dominant male meerkats. Molecular Ecology, 17, 2287-2299.

Staub, N.L. \& DeBeer, M. 1997. The role of androgens in female vertebrates. General and Comparative Endocrinology, 108, 1-24.

Trivers, R.L. \& Willard, D.E. 1973. Natural selection of parental ability to vary sex-ratio of offspring. Science, 179, 90-92.

Uller, T., Pen, I., Wapstra, E., Beukeboom, L.W. \& Komdeur, J. 2007. The evolution of sex ratios and sex-determining systems. Trends in Ecology \& Evolution, 22, 292-297. Vandenbergh, J. \& Huggett, C.L. 1994. Mothers prior intrauterine position affects the sex ratio of her offspring in house mice. Proceedings of the National Academy of Sciences, U.S.A., 91, 11055-11059.

Wachter, B., Höner, O.P., East, M.L., Golla, W. \& Hofer, H. 2002. Low aggression levels and unbiased sex ratios in a prey-rich environment: no evidence of siblicide in Ngorongoro spotted hyenas (Crocuta crocuta). Behavioral Ecology and Sociobiology, 52, 348-356. 
West, S.A., Reece, S.E. \& Sheldon, B.C. 2002. Sex ratios. Heredity, 88, 117-124.

Williams, G.C. 1979. Question of adaptive sex-ratio in outcrossed vertebrates. Proceedings of the Royal Society B, 205, 567-580.

Young A. J. 2003. Subordinate tactics in cooperative meerkats: helping, breeding and dispersal. Ph.D. thesis, University of Cambridge.

Young, A.J. \& Clutton-Brock, T.H. 2006. Infanticide by subordinates influences reproductive sharing in cooperatively breeding meerkats. Biology Letters, 2, 385-387. 